FERMILAB-FN-0762-AD

\title{
Comments on Landau Damping due to Synchrotron Frequency Spread
}

\author{
K.Y. Ng \\ Fermi National Accelerator Laboratory, ${ }^{*}$ P.O. Box 500, Batavia, IL 60510 \\ (January 1, 2005)
}

\begin{abstract}
An inductive/space-charge impedance shifts the synchrotron frequency downwards above/below transition, but it is often said that the coherent synchrotron frequency of the bunch is not shifted in the rigid-dipole mode. On the other hand, the incoherent synchrotron frequency due to the sinusoidal rf always spreads in the downward direction. This spread will therefore not be able to cover the coherent synchrotron frequency, implying that there will not be any Landau damping no matter how large the frequency spread is. By studying the dispersion relation, it is shown that the above argument is incorrect, and there will be Landau damping if there is sufficient frequency spread. The main reason is that the coherent frequency of the rigid-dipole mode will no longer remain unshifted in the presence of a synchrotron frequency spread.
\end{abstract}

${ }^{*}$ Operated by the Universities Research Association, Inc., under contract with the U.S. Department of Energy. 


\section{Introduction}

A capacitive or space-charge impedance below transition (or an inductive impedance above transition) shifts the incoherent synchrotron frequency downwards while it is often said that the coherent synchrotron frequency of the rigid-dipole mode of the bunch is not shifted and remains at the value of the bare synchrotron frequency. Let us turn on synchrotron frequency spread as a result of the sinusoidal rf potential. This provides the incoherent synchrotron frequency a spread also in the downwards direction. Thus the coherent synchrotron frequency appears always to be not covered by the incoherent spread at all, and therefore there would not be any Landau damping no matter how large the frequency spread is. An illustration is shown in Fig. 1. The figure is drawn schematically for the Tevatron in Run II $36 \times 36$

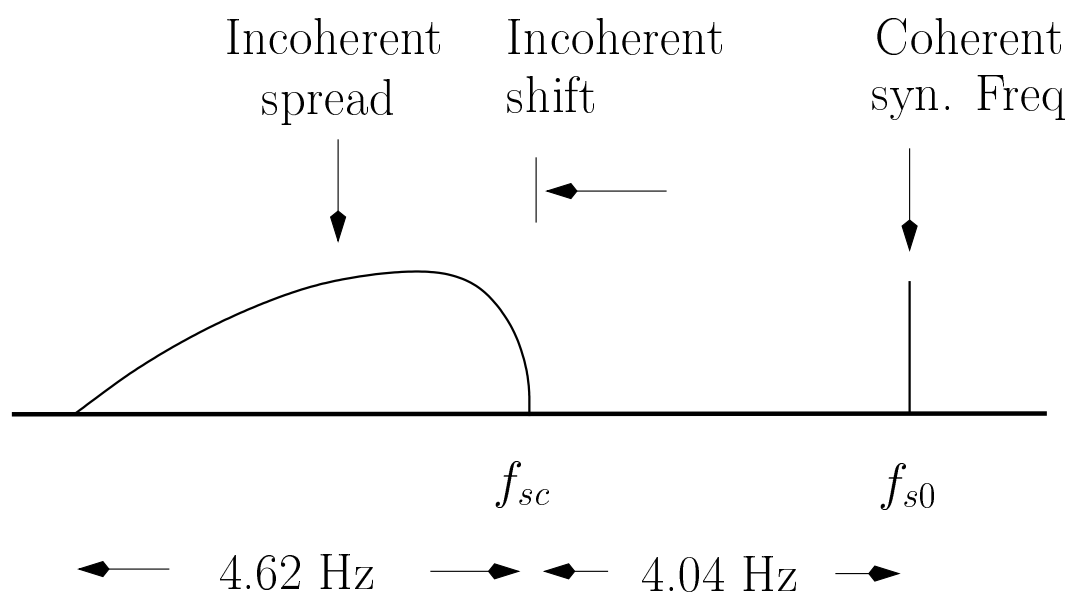

Figure 1: Schematic drawing showing the incoherent spread of of the synchrotron frequency in the sinusoidal rf potential shifted away from the coherent synchrotron frequency which remains at the bare frequency $f_{s 0}$, thus not being able to provide Landau damping. Here $f_{s c}$ denotes the incoherent synchrotron frequency of the particles at the center of the bunch. The figure is drawn schematically for the Tevatron in Run II $36 \times 36$ operation.

operation, where the proton bunch has the intensity $N_{b}=2.7 \times 10^{10}$ with a rms bunch length $\sigma_{\ell}=37 \mathrm{~cm}$. At injection, the energy is $E_{0}=150 \mathrm{GeV}$. Assuming a parabolic distribution with half-bunch length $\hat{\tau}=\sqrt{5} \sigma_{\ell} /(\beta c)$, the incoherent synchrotron frequency shift subject to an inductive longitudinal impedance $\operatorname{Im} Z_{0}^{\|}=3 \Omega$ is given by

$$
\left.\frac{\Delta f_{s}}{f_{s 0}}\right|_{\text {incoh }}=-\frac{3 \pi I_{b} \mathcal{I} m Z_{0}^{\|} / n}{2 h V_{\text {rf } \cos \phi_{s}\left(\hat{\tau} \omega_{0}\right)^{3}}}=-0.0463 \quad \text { or }\left.\quad \Delta f_{s}\right|_{\text {incoh }}=-4.04 \mathrm{~Hz} \text {, }
$$


where use has been made of the mean ring radius $R=1 \mathrm{~km}$, the rf harmonic $h=1113$, the rf voltage $V_{\mathrm{rf}}=1 \mathrm{MV}$, the rf synchronous phase $\phi_{s}=\pi$, the slip factor $\eta=+0.002827$, the bare synchrotron frequency $f_{s 0}=f_{0} \sqrt{-\eta h e V_{\mathrm{rf}} \cos \phi_{s} /\left(2 \pi \beta^{2} E_{0}\right)}=87.2 \mathrm{~Hz}$, and the average bunch current $I_{b}=e N_{b} f_{0}$ with $f_{0}=\omega_{0} /(2 \pi)=47.7 \mathrm{kHz}$ denoting the revolution frequency. The spread of the synchrotron frequency from the center to the edge of the bunch can be approximated by [1]

$$
\left.\frac{\Delta f_{s}}{f_{s 0}}\right|_{\text {spread }}=\left(\frac{1+\sin ^{2} \phi_{s}}{1-\sin ^{2} \phi_{s}}\right)\left(\frac{\pi h \hat{\tau} f_{0}}{2}\right)^{2}=0.0529 \quad \text { or }\left.\quad \Delta f_{s}\right|_{\text {spread }}=-4.62 \mathrm{~Hz} .
$$

The purpose of this paper is to demonstrate that the above assertion is incorrect, and there will be Landau damping provided that the spread in incoherent synchrotron frequency is large enough. The main reason is that the coherent synchrotron frequency will not remain unshifted in the presence of the synchrotron frequency spread. The investigation is through the dispersion relation of a bunched beam, which will be reviewed in Sec. 2. The elliptical distribution in the longitudinal phase space (or parabolic linear distribution along the phase or time axis) will be used in the study. As an addendum, we are showing also stability threshold curves for other distributions and also in the higher azimuthal modes.

\section{Dispersion Relation}

The discussions on collective instability and Landau damping are best derived from the dispersion relation, for which we are going to give a brief derivation. $[1,2]$ Let $\tau$ be the arrival time of a particle at a location in the ring ahead of the synchronous particle, and $p_{\tau}=\eta \Delta E /\left(\beta^{2} \omega_{s c} E_{0}\right)$ its canonical momentum. They obey the equations of motion:

$$
\left\{\begin{aligned}
\frac{d \tau}{d s} & =-\frac{\omega_{s c}}{v} p_{\tau} \\
\frac{d p_{\tau}}{d s} & =\frac{\omega_{s c}}{v} \tau+\frac{\eta}{E_{0} \omega_{s c} \beta^{2}}\left\langle F_{0}^{\|}(\tau ; s)\right\rangle_{\mathrm{dyn}},
\end{aligned}\right.
$$

where $\omega_{s c}$ is the incoherent angular synchrotron frequency of the particles at the center of the bunch and $v=\beta c$ is the nominal velocity. This formulation makes the two canonical variables more symmetric. In the absence of the wake force $\left\langle F_{0}^{\|}(\tau ; s)\right\rangle_{\mathrm{dyn}}$, the trajectory of a beam particle is just a circle in the longitudinal phase space. In above, the dynamic part of the longitudinal wake force is defined as

$$
\left\langle F_{0}^{\|}(\tau ; s)\right\rangle_{\mathrm{dyn}}=\left\langle F_{0}^{\|}(\tau ; s)\right\rangle_{\text {total }}-\left\langle F_{0}^{\|}(\tau ; s)\right\rangle_{\text {stat }},
$$


and only the dynamic or time-dependent part of the linear density of the bunch will contribute. The static part of the linear density has already been taken care of by solving the problem of potential-well distortion so that we have the incoherent synchrotron frequency $\omega_{s c}$ used in the equations of motion and the Vlasov equation below instead of the bare synchrotron frequency $\omega_{s 0}$.

The phase-space distribution $\psi$ of a bunch can be separated into the unperturbed or stationary part $\psi_{0}$ and the perturbed part $\psi_{1}$ :

$$
\psi(\tau, \Delta E ; s)=\psi_{0}(\tau, \Delta E)+\psi_{1}(\tau, \Delta E ; s)
$$

where $\psi_{0}(\tau \Delta E)$ is obtained from solving the problem of potential-well distortion in the zeroth order Vlasov equation. The first order Vlasov equation is

$$
\frac{\partial \psi_{1}}{\partial s}-\frac{\omega_{s c}}{v} p_{x} \frac{\partial \psi_{1}}{\partial x}+\frac{\omega_{s c}}{v} x \frac{\partial \psi_{1}}{\partial p_{x}}+\frac{\partial \psi_{0}}{\partial p_{x}} \frac{\eta}{E_{0} \omega_{s c} \beta^{2}}\left\langle F_{0}^{\|}(\tau ; s)\right\rangle_{\mathrm{dyn}}=0
$$

and, in the circular coordinates,

$$
\left\{\begin{aligned}
\tau & =r \cos \phi \\
p_{\tau} & =r \sin \phi
\end{aligned}\right.
$$

it simplifies to

$$
\frac{\partial \psi_{1}}{\partial s}+\frac{\omega_{s c}}{v} \frac{\partial \psi_{1}}{\partial \phi}+\frac{\eta}{E_{0} \omega_{s c} \beta^{2}} \frac{d \psi_{0}}{d r} \sin \phi\left\langle F_{0}^{\|}(\tau ; s)\right\rangle_{\mathrm{dyn}}=0
$$

The perturbed distribution can be expanded azimuthally in the longitudinal phase space,

$$
\psi_{1}(r, \phi ; s)=\sum_{m} \alpha_{m} R_{m}(r) e^{i m \phi-i \Omega s / v}
$$

where $R_{m}(r)$ are functions corresponding to the $m$ th azimuthal, $\alpha_{m}$ are the expansion coefficients, and $\Omega /(2 \pi)$ is the collective frequency to be determined. In above, $m=0$ has been excluded because it has been included in the stationary part $\psi_{0}$, otherwise charge conservation will be violated. The Vlasov equation becomes

$$
\left(\Omega-m \omega_{s c}\right) \alpha_{m} R_{m}(r) e^{-i \Omega s / v}=-\frac{i v \eta}{E_{0} \omega_{s c} \beta^{2}} \frac{d \psi_{0}}{d r} \int_{-\pi}^{\pi} \frac{d \phi}{2 \pi} e^{-i m \phi} \sin \phi\left\langle F_{0}^{\|}(\tau ; s)\right\rangle_{\mathrm{dyn}} .
$$

The wake force acting on a beam particle at location $s$, with time advance $\tau$ relative to the synchronous particle, due to all preceding particles passing through $s$ earlier can be expressed as

$$
\left\langle F_{0}^{\|}(\tau ; s)\right\rangle_{\mathrm{dyn}}=-\frac{e^{2}}{2 \pi R} \int_{-\infty}^{\infty} d \tau^{\prime} K\left(\tau^{\prime}-\tau\right) \lambda_{1}\left(\tau^{\prime} ; s\right)
$$


where the translational-invariant kernel $K\left(\tau^{\prime}-\tau\right)$ is exact for continuous interactions such as space-charge or resistive-wall (assuming smooth walls), and retains the average effect of localized structures such as cavities. For a broadband impedance the kernel is the same as the longitudinal monopole wake potential, or $K(\tau)=W_{0}^{\prime}(\tau)$. Since the perturbed linear distribution $\lambda_{1}(\tau ; s)$ is equal to the projection of $\psi_{1}(\tau, \Delta E ; s)$ onto the $\tau$-axis, Eq. (2.8) becomes an eigen-equation in the $R_{m}(r)$ after substituting the azimuthal expansion of Eq. (2.7).

We now make the approximation that the perturbation is small so that $\Omega-m \omega_{s c} \ll$ $\omega_{s c}$, implying that the coupling between different azimuthal modes can be neglected. This simplifies the Vlasov equation to one involving only one azimuthal mode $m$,

$$
\left(\Omega-m \omega_{s c}\right) R_{m}(r)=-\frac{d \phi_{0}}{d r} \int_{0}^{\infty} G_{m}\left(r, r^{\prime}\right) R_{m}\left(r^{\prime}\right) r^{\prime} d r^{\prime},
$$

where the interaction matrix is

$$
G_{m}\left(r, r^{\prime}\right)=-\frac{i e^{2} v}{2 \pi C} \int_{0}^{2 \pi} d \phi \int_{0}^{2 \pi} d \phi^{\prime} K\left(r^{\prime} \cos \phi^{\prime}-r \cos \phi\right) \sin \phi e^{i m\left(\phi^{\prime}-\phi\right)} .
$$

Equation (2.10) is the simplified form of the Sacherer's integral equation. [3] Given a longitudinal wake $W_{0}^{\|}(\tau)$ and an unperturbed distribution $\psi_{0}(r)$, the coherent frequency of excitation $\Omega$ can be solved. Unfortunately, analytic solutions are only possible for some particular $\psi_{0}(r)$, especially when there is a spread in the incoherent synchrotron frequency, such as inside an rf bucket. In order to extract some useful information concerning the stability of the particle beam, we resort to the method of synthetic kernel, which involves the substitution of the kernel of longitudinal dipole wake by a simple function. To study dipole motion, we assume the wake force to be proportional to the longitudinal position of the bunch center, an assumption which should be true only when the bunch is rigid. Looking at Eq. (2.9), we can readily set the kernel as

$$
K\left(\tau^{\prime}-\tau\right)=A\left(\tau^{\prime}-\tau\right)=A\left(r^{\prime} \cos \phi^{\prime}-r \cos \phi\right)
$$

where $A$ is a constant. We obtain immediately

$$
G_{m}\left(r, r^{\prime}\right)=-\frac{\pi e^{2} v A}{2} r^{\prime} \delta_{m 1}
$$

or only the $m=1$ dipole mode will contribute as expected. Substitution into Eq. (2.10) gives

$$
R_{1}(r)=\frac{\pi e^{2} v A}{2} C_{1} \frac{\psi_{0}^{\prime}(r)}{\Omega-\omega_{s c}}
$$


where the prime denotes derivative with respect to $r$ and

$$
C_{1}=\int_{0}^{\infty} R_{1}\left(r^{\prime}\right) r^{\prime 2} d r^{\prime}
$$

is a constant. Landau damping is introduced by assuming a spread in the incoherent synchrotron frequency, making it a function of $r$ such that $\omega_{s}(0)=\omega_{s c}$. Thus multiplying both sides by $r^{2}$ and integrating over $r$ leads to the dispersion relation

$$
1=-\pi \Delta \omega_{s \mathrm{dyn}} \int_{0}^{\infty} \frac{\psi_{0}^{\prime}(r) r^{2}}{\Omega-\omega_{s}(r)} d r
$$

where $\Delta \omega_{s \text { dyn }}=-e^{2} v A / 2$. Actually $\Delta \omega_{s \text { dyn }}$ has a physical meaning. When there is no spread in the incoherent synchrotron frequency, the denominator of the integrand can be moved to the left side. It is then easy to verify that $\Delta \omega_{s \text { dyn }}=\Omega-\omega_{s c}$, or $\Delta \omega_{s \text { dyn }}$ is the dynamical shifting of the incoherent synchrotron frequency $\omega_{s \text { dyn }}$ towards the coherent frequency $\Omega$. Since the incoherent synchrotron frequency shift for particles at the bunch center is defined as

$$
\Delta \omega_{s \text { incoh }}=\omega_{s c}-\omega_{s 0}
$$

we arrive at

$$
\Delta \omega_{\text {syn }}=\left(\Omega-\omega_{s 0}\right)-\Delta \omega_{s \text { incoh }},
$$

implying that $\Delta \omega_{s \text { dyn }}$ is the difference between the coherent frequency shift and incoherent frequency shift. We refer $\Delta \omega_{s \text { dyn }}$ as the dynamic frequency shift arriving from the dynamic effects of the wake fields. As an example, let us consider the elliptical distribution

$$
\psi_{0}(r)=\frac{3}{2 \pi \hat{\tau}^{2}} \sqrt{1-\frac{r^{2}}{\hat{\tau}^{2}}},
$$

when $r<\hat{\tau}$ and zero otherwise. When projected onto the $\tau$-axis, this distribution leads to the parabolic linear distribution

$$
\lambda_{0}(\tau)=\frac{3}{4 \hat{\tau}}\left(1-\frac{\tau^{2}}{\hat{\tau}^{2}}\right),
$$

when $|\tau|<\hat{\tau}$ and zero otherwise. Let us first neglect the synchrotron frequency spread in the sinusoidal rf potential. In the presence of a constant $\mathcal{I} m Z_{0}^{\|} / n$ such as space-charge below transition, every particle in the bunch including the one at the bunch center has the same self-force frequency shift given by Eq. (1.1), which can also be rewritten as

$$
\Delta \omega_{\text {sincoh }}=\frac{3 e^{2} N_{b} \eta \mathcal{I} m Z_{0}^{\|} / n}{8 \pi \beta^{2} E_{0} \omega_{s 0} \hat{\tau}^{3}} .
$$


Notice that this frequency shift is negative when driven by a space-charge impedance below transition or inductive above transition. Now because of the dynamic effects of the wake fields, the coherent frequency at which the center of the bunch is oscillating receives an additional dynamical shift $\Delta \omega_{s \text { dyn }}$. Thus the coherent synchrotron frequency of the bunch is

$$
\Omega=\omega_{s 0}+\Delta \omega_{s \text { incoh }}+\Delta \omega_{s \text { dyn }} .
$$

Because the wake-field pattern and therefore the potential-well distortion moves with the bunch in this rigid-dipole mode, the bunch center will not be affected by the wake field at all. For this reason, we must have in this case the cancellation of the dynamic frequency shift and the incoherent frequency shift so that the coherent frequency $\Omega$ is just equal to the bare synchrotron frequency. As will be demonstrated below, however, this is true only when there is no spread in the incoherent synchrotron frequency.

Before ending this section, let us give some more comments about how the synthetic kernel has affected the problem physically. There is only one rigid-dipole mode (with $m=1$ ), which is generated by displacing the unperturbed bunch distribution $\psi_{0}$ from the equilibrium position of the rf potential so that the distribution rotates rigidly with the synchrotron frequency. However, there are infinite number of nonrigid dipole modes with $m=1$. For example, we can imagine the bunch as an elastic sheet in the longitudinal phase space and has only its center part displaced from the equilibrium position of the rf potential but leaving the edge part of the distribution undisturbed. The perturbation now rotates with synchrotron frequency but with a particular distribution radially. The same applies to the quadrupole modes $(m=2)$, sextupole modes $(m=3)$, etc. In other words, there are infinite number of radial modes associated with each azimuthal mode. The synthetic kernel just singles out the most easily excited radial mode (the one with the least frequency shift) for each azimuthal, thus reducing the twofold infinity of modes to only one series of modes.

\section{Elliptical Distribution}

In the elliptical distribution, the only spread of incoherent synchrotron frequency comes from the sinusoidal rf potential well. With small half bunch length $\hat{\tau}$, this spread is given by

$$
\omega_{s \operatorname{incoh}}(\hat{\tau})=\omega_{s c}-S \hat{\tau}^{2}
$$


where $\omega_{s c}$ is the incoherent angular synchrotron frequency of particles at the center of the bunch and the spread $S$ can be can be read off from Eq. (1.2),

$$
S=\left(\frac{h \omega_{0}}{4}\right)^{2} \frac{1+\sin ^{2} \phi_{s}}{1-\sin ^{2} \phi_{s}}
$$

where $h$ is the rf harmonic. The dependence on the synchronous angle $\phi_{s}$ is the result of a fitting with numerical computation. Here, the expression for $S$, quoted for the sake of completeness, will not be used in the discussion below. However, it is important to point out that this spread of synchrotron frequency, as defined in Eq. (3.1) is measured from $\omega_{s c}$, the incoherent synchrotron frequency of the particles at the center of the bunch.

For the rigid-dipole mode $(m=1)$, the dispersion relation now takes the form

$$
1=-\pi \Delta \omega_{s \text { dyn }} \int_{0}^{\infty} \frac{\psi_{0}^{\prime}(r) r^{2}}{\Omega-\omega_{s c}+S r^{2}} d r .
$$

Let us introduce the dimensionless variable

$$
z=\frac{\omega_{s c}-\Omega}{S}
$$

and change the variable of integration to $t=r^{2}$. The dispersion relation becomes

$$
1=-\frac{\pi \Delta \omega_{s \mathrm{dyn}}}{S} \int_{0}^{\infty} \frac{\psi_{0}^{\prime}(t) t}{t-z+i \epsilon} d t,
$$

where $\psi_{0}^{\prime}(t)=d \psi_{0}(r) /\left.d r^{2}\right|_{r^{2}=t}$, and an infinitesimal imaginary part $i \epsilon$ with $\epsilon>0$ has been added to represent the situation just above threshold when $z$ is real. Now the explicit expression for the elliptical distribution, Eq. (2.19), is substituted and the dispersion integral is evaluated to arrive at the threshold curve:

$$
\left(\frac{\Delta \omega_{s \mathrm{dyn}}}{S}\right)^{-1}= \begin{cases}\frac{3}{4}\left[2+\frac{z}{\sqrt{1-z}} \ln \frac{\sqrt{1-z}+1}{\sqrt{1-z}-1}\right], & z<0, \\ \frac{3}{4}\left[2+\frac{z}{\sqrt{1-z}} \ln \frac{1+\sqrt{1-z}}{1-\sqrt{1-z}}-i \pi \frac{z}{\sqrt{1-z}}\right], & 0<z<1, \\ \frac{3}{4}\left[2-\frac{2 z}{\sqrt{z-1}} \tan ^{-1} \frac{1}{\sqrt{z-1}}\right], & 1<z,\end{cases}
$$

where, for the sake of convenience, we have set the half bunch length to $\hat{\tau}=1$. The stability threshold curve is depicted in the complex $\left(\Delta \omega_{s \text { dyn }} / S\right)$-plane in Fig. 2. We have plotted $\Delta \omega_{s \text { dyn }} / S$ as a function of $z$ in Fig. 3 to facilitate our discussion below. As defined in the previous section, $\operatorname{Re} \Delta \omega_{s \text { dyn }}$ is the dynamic part of the coherent synchrotron tune shift of the 


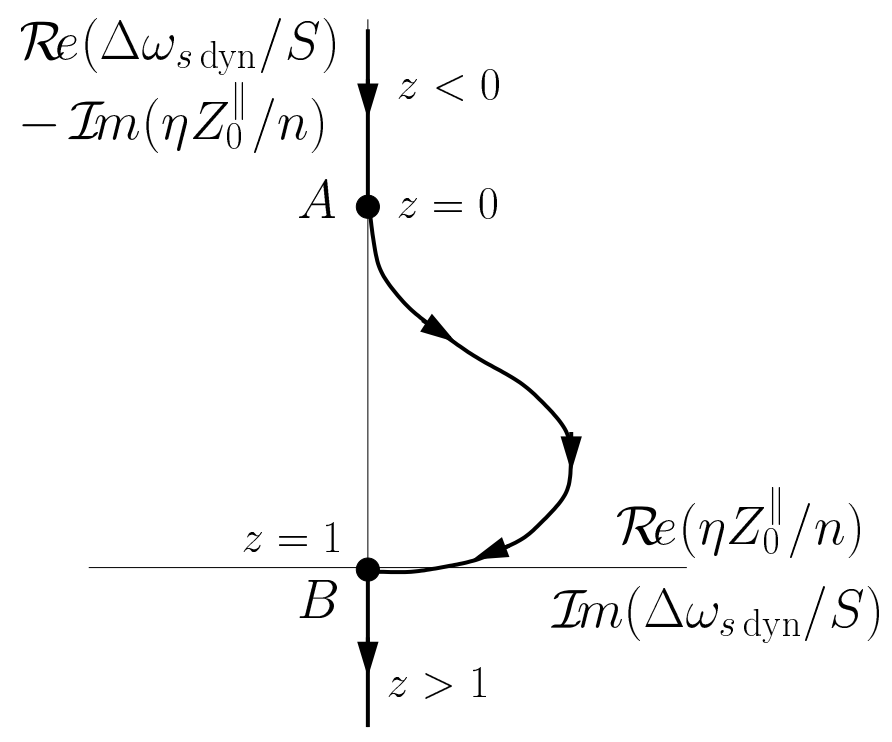

Figure 2: Stability threshold curve for elliptical distribution. The arrows point to the direction of increasing $z$ (decreasing $\Omega$ ).

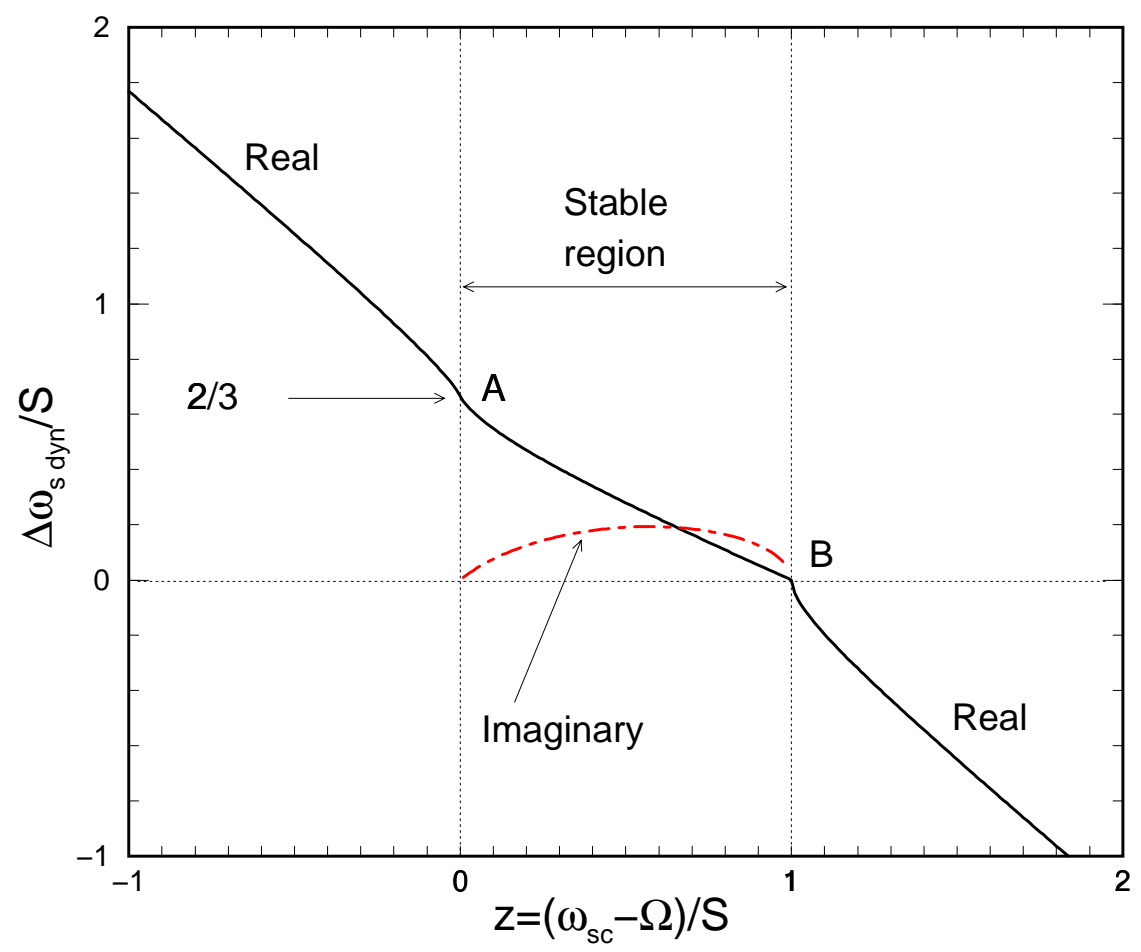

Figure 3: $\Delta \omega_{s \text { dyn }} / S$ as a function of $z=\left(\omega_{s c}-\Omega\right) / S$ in the stability threshold contour. 
bunch in the absence of synchrotron frequency spread $(S=0)$. It is equal to the incoherent synchrotron frequency shift $\Delta \omega_{\text {sincoh }}$ and is therefore a measure of the reactive impedance per harmonic of the vacuum chamber. When $z$ is negative, the dynamic part of the coherent frequency shift $\Delta^{\dagger} \omega_{s \text { coh }}^{\text {dyn }}=\Omega-\omega_{s c}$ is real and positive in the presence of synchrotron frequency spread $(S>0)$ when measured from the frequency $\omega_{s c}$. Thus, the coherent synchrotron frequency is away from the incoherent synchrotron frequency spread. There is no Landau damping and the bunch is unstable in the presence of any small $\operatorname{Re} Z_{0}^{\|}$. When $z=0$, there is no dynamic coherent frequency shift or the coherent synchrotron frequency is right at $\omega_{s c}$, just at the edge of stability. In the plot, this corresponds to $\Delta \omega_{s \text { dyn }} / S=\frac{2}{3}$ or Point A. As $z$ turns positive, the dynamic coherent synchrotron frequency shift $\Delta \omega_{s \text { coh }}^{\text {dyn }}$ becomes negative when measured from $\omega_{s c}$. The beam is now inside the stability region. This is the region between Points A and B in stability plot. As the reactive impedance of the vacuum chamber becomes smaller and smaller $\left(\Delta \omega_{s \text { dyn }} / S \rightarrow 0\right)$, Point B is approached with $z \rightarrow 1-$. When the reactive impedance changes $\operatorname{sign}\left(\eta \mathcal{I} m Z_{0}^{\|} / n\right.$ going from negative to positive), $z>1$, Landau damping vanishes and the beam becomes unstable again.

The corresponding linear density of the particle bunch as a function of synchrotron frequency is shown schematically in Fig. 4. The top plot is for the situation when $\eta \mathcal{I} m Z_{0}^{\|} / n<0$. In this plot, when $\Delta \omega_{s \text { coh }}^{\text {dyn }}=-0.26 S(z=0.26)$, Re $\Delta \omega_{s \text { dyn }}=-\Delta \omega_{s \text { incoh }}=0.429 S$ and Im $\Delta \omega_{s \text { dyn }}=0.146 S$ on the stability contour, which are obtained by solving the dispersion relation or Eq. (3.6). The coherent synchrotron frequency is depicted by the vertical arrows between Points A and B. We see that the coherent synchrotron frequency now lies inside the incoherent frequency spread which provides Landau damping. Of course, the beam will become unstable if $\mathcal{I} m \Delta \omega_{s \text { dyn }}>0.146 S$ (or $\operatorname{Re} Z_{0}^{\|}$becomes larger) and $z$ will have a negative imaginary part $(\Omega$ will have a positive imaginary part). Let us imagine that the incoherent frequency spread $S$ is getting smaller and smaller while $\Delta \omega_{\text {sincoh }}$ is held fixed. In another words, $\operatorname{Re} \Delta \omega_{s \mathrm{dyn}} / S$ becomes larger and larger. From Fig. 3, $z$ decreases or the dynamic coherent synchrotron frequency shift $\Delta \omega_{s \text { coh }}^{\text {dyn }}$ becomes less negative until it reaches zero $(z=0)$ arriving at Point A when $S=\frac{3}{2} \Delta \omega_{s \text { dyn }}=-\frac{3}{2} \Delta \omega_{\text {sincoh. As }} S$ continues to shrink, $\Delta \omega_{s \text { coh }}^{\text {dyn }}$ becomes positive and goes outside the incoherent frequency spread. As $S \rightarrow 0$, we

\footnotetext{
${ }^{\dagger}$ Here, $\Delta \omega_{s \text { coh }}^{\text {dyn }}$ is the dynamic part of the coherent shift and is measured from $\omega_{s c}$. The total coherent shift is measured from the bare frequency $\omega_{s 0}$ and contains, in addition to this dynamical part, a static part which is equal to the incoherent shift $\Delta \omega_{s}$ incoh $=\omega_{s c}-\omega_{s 0}$. On the other hand $\Delta \omega_{s}$ dyn represents the same dynamic part of the coherent shift measured from $\omega_{s c}$ only in the absence of incoherent synchrotron frequency spread.
} 

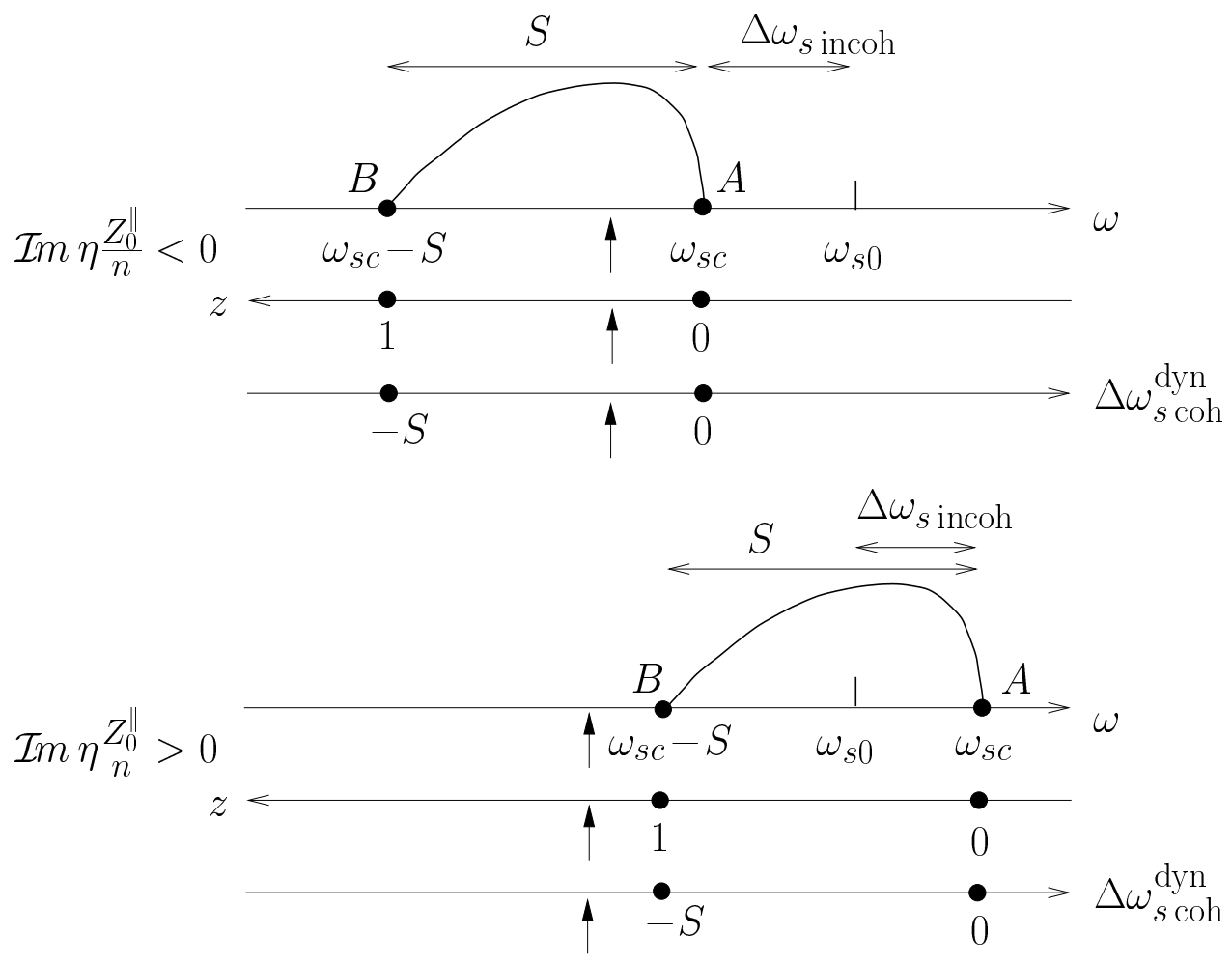

Figure 4: Plot of linear bunch density as functions of synchrotron frequency $\omega, z$, and the dynamical part of the coherent synchrotron frequency shift $\Delta \omega_{s \text { coh }}^{\text {dyn }}$ when (top) $\eta \mathcal{I} m Z_{0}^{\|} / n<0$ and (bottom) $\eta \operatorname{Im} Z_{0}^{\|} / n>0$. The vertical arrows point to the coherent synchrotron frequency of the bunch. Thus, when $\operatorname{Re} Z_{0}^{\|}$is small, the bunch is stable in the top plot, but unstable in the bottom plot. Points A and B correspond to the same Points A and B in the threshold plot of Fig. 2.

know from Fig. 3 and Eq. (3.6) that $z \rightarrow-\infty$. Solving Eqs. (3.4) and (3.6), one finds $\Delta \omega_{s \text { coh }}^{\mathrm{dyn}} \rightarrow-\Delta \omega_{s \text { inoch }}$ or the coherent synchrotron frequency goes back to the unperturbed value of $\omega_{s 0}$ as expected. On the other hand, we can fix the incoherent frequency spread $S$ and reduce $\Delta \omega_{s c}$ or $\mathcal{R e} \omega_{s \text { dyn }}$ instead until it reaches zero. The coherent synchrotron frequency will shift towards Point B. It reaches Point B when $\Delta \omega_{\text {sincoh }}$ reaches zero $(z$ reaches $+1)$. Thus, when $\eta \operatorname{Im} Z_{0}^{\|} / n<0, z$ varies from $-\infty$ to +1 .

The bottom plot of Fig. 4 is for the situation when $\eta \operatorname{Im} Z_{0}^{\|} / n>0$. Now the incoherent frequency shift $\Delta \omega_{\text {sincoh }}$ in the absence of incoherent frequency spread becomes positive and the incoherent frequency spread may encompass the unperturbed synchrotron frequency $\omega_{s 0}$ as illustrated in the plot. However, there is no Landau damping, because under this situation

\footnotetext{
${ }^{\ddagger}$ As $z \rightarrow-\infty$, Eq. (3.6) becomes $\Delta \omega_{s}$ dyn $/ S \rightarrow 1-z$. With $z=-\Delta \omega_{s \text { coh }}^{\text {dyn }} / S$, we obtain $\Delta \omega_{s \text { coh }}^{\text {dyn }} \rightarrow$ $\Delta \omega_{s \text { dyn }}-S \rightarrow-\Delta \omega_{s \text { incoh }}$
} 
$z>1$ (see Fig. 3 when $\Delta \omega_{s \text { dyn }}=-\Delta \omega_{\text {sincoh }}<0$ ). In other words, the dynamic coherent synchrotron frequency shifts negatively from $\omega_{s c}$ and the shift must be larger than $\omega_{s c}-S$, which is towards the right side of Point B. This plot shows $\Delta \omega_{s \text { dyn }}=-0.40 S$. Solution of Eq. (3.6) gives $z=1.27$. This value of coherent synchrotron frequency is depicted by the vertical arrows. It is easy to see that no matter how large or small the incoherent frequency shift is, the coherent synchrotron frequency is always outside the incoherent frequency spread between Points $\mathrm{A}$ and $\mathrm{B}$, or the beam is always unstable independent of how small $\operatorname{Re} Z_{0}^{\|}$is.

\section{Equi-Growth Contours}

With a purely inductive wall above transition or a purely capacitive space-charge force below transition, the incoherent synchrotron frequency shift $\Delta \omega_{\text {sincoh }}$ in an elliptical distribution without any frequency spread from the nonlinearity of the rf potential is given by Eq. (2.21) and is negative. We have shown in the above that this is equal to the negative of $\operatorname{Re} \Delta \omega_{s \text { dyn }}$, real part of the dynamical frequency shift (in the absence of incoherent synchrotron frequency spread). It is easy to understand that the incoherent frequency shift in the absence of frequency spread is always real even when the coupling impedance contains a real part. This is because the incoherent frequency shift is obtained by linearizing the driving force $\left\langle F_{0}^{\|}(\tau ; s)\right\rangle_{\text {dyn }}$ on the right-side of Eq. (2.1), which consists of the convolution of the wake potential and the linear beam distribution and both of them are real. However, $\Delta \omega_{s \text { dyn }}$ as defined in Eq. (3.3) is in general, complex. Since $\operatorname{Re} \Delta \omega_{s \text { dyn }}$ is well-defined and is proportional to $\operatorname{Im} Z_{0}^{\|} / n, \Delta \omega_{s \text { dyn }}$, being analytic in frequency can be defined easily by analytic continuation. In other words, we can write

$$
\Delta \omega_{\text {syn }} \sim-\operatorname{Im}(\eta Z) \longrightarrow-\operatorname{Im}(\eta Z)+i \operatorname{Re}(\eta Z)
$$

This explains why $\operatorname{Re}(\eta Z)$ and $\operatorname{Im}(\Delta \omega / S)$ are in the same direction in the left plot of Fig. 2, while $\operatorname{Im}(\eta Z)$ and $\operatorname{Re}(\Delta \omega / S)$ are in the opposite directions. Thus, in the absence of incoherent frequency spread,

$$
\operatorname{Im} \Omega=\frac{3 c^{2} N_{b}}{8 \pi \beta^{2} E_{0} \omega_{s 0} \hat{\tau}^{3}}\left(\eta \frac{\mathcal{R e} Z_{0}^{\|}}{n}\right),
$$

and the solution is unstable/stable depending on $\eta \mathcal{R} e Z_{0}^{\|} / n \gtrless 0$. Therefore in the $\left(\Delta \omega_{s \text { dyn }} / S\right)$ space such as in the left plot of Fig. 2, the left half is stable and the right half unstable in 

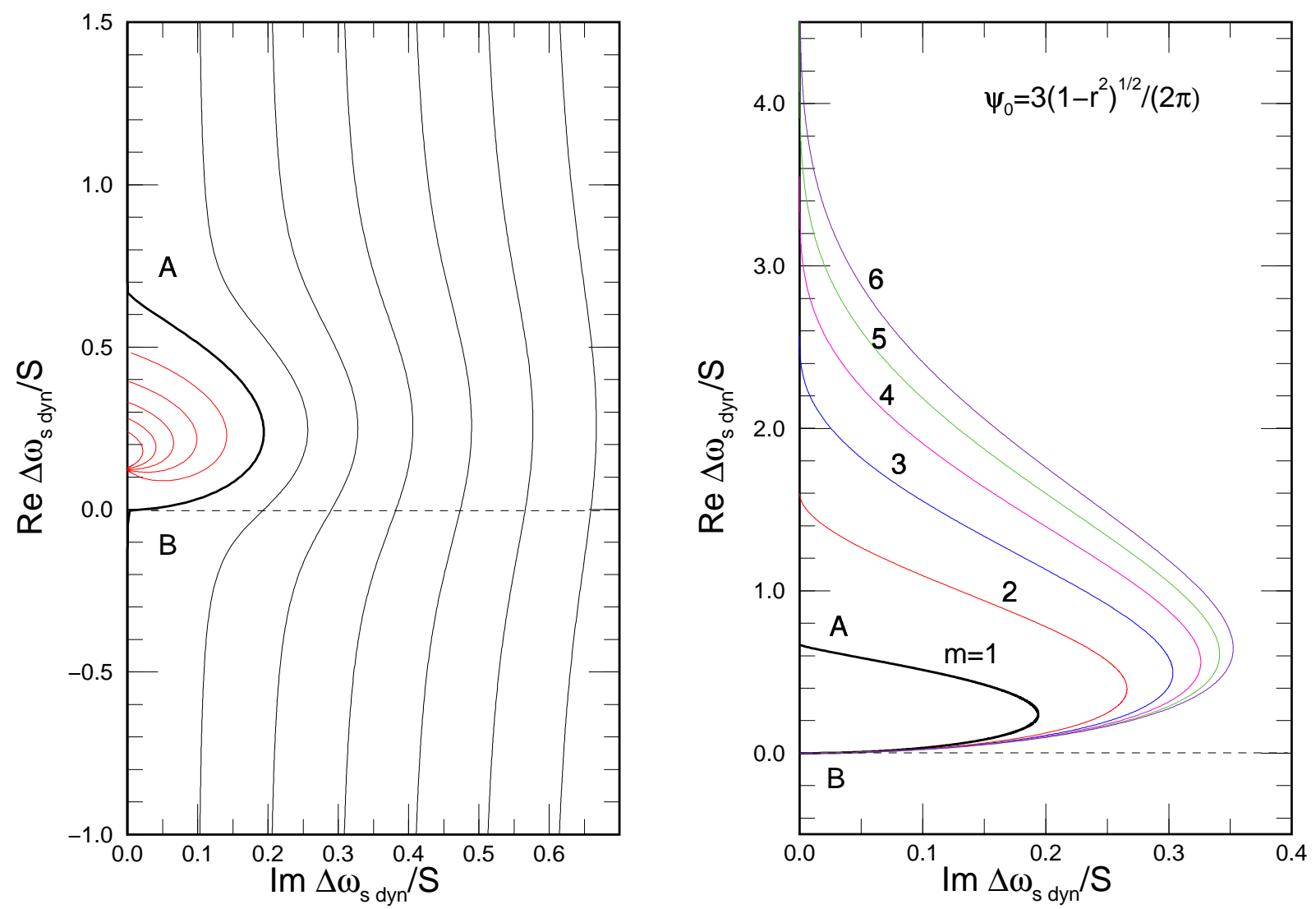

Figure 5: Left: Equi-Growth contours of the elliptical distribution $\frac{3}{2 \pi} \sqrt{1-r^{2}}$, with growth rate increasing by steps of $0.1 S$ to the left of the stability contour (darker curve) and damping rate increasing by steps of $0.1 S$ to the right. Right: Stability contours for the elliptical distribution for azimuthal $m=1$ to 6 .

the absence of incoherent synchrotron frequency spread, and the stability contour is just the $\operatorname{Re} \Delta \omega_{s \mathrm{dyn}} / S$ coordinate axis. when there is finite incoherent synchrotron frequency spread, The stability contour is pushed to the right so that a solution with $\eta \operatorname{Re} Z_{0}^{\|} / n>0$ can still be stable provided that it falls to the left-side of the stability contour. The equi-growth contours and equi-damping contours can be easily computed by letting $z$ complex in Eq. (3.6). The result is shown in the left plot of Fig. 5. The thick contour is the stability contour. To the right, the equi-growth contours correspond to growth rates at steps of $0.1 S$. To the left, the equi-damping contours correspond to damping rates at steps of $-0.1 S$. It is clear that Points $\mathrm{A}$ and $\mathrm{B}(z=0$ and 1$)$ are two branch points and the straight line $\mathrm{AB}$ is a branch cut. 


\section{$5 \quad$ Stability of a Bunch}

The upper and lower synchrotron sidebands flanking a positive revolution harmonic correspond to the upper sidebands associated with, respectively, the positive and negative revolution harmonics. Since $\operatorname{Re} Z_{0}^{\|} / n$ is an odd function of frequency, $\eta \mathcal{R} e Z_{0}^{\|} / n$ is positive for the upper sidebands and negative for the lower sidebands above transition $(\eta>0)$. As a result, all the upper sidebands are stable, while all the lower sidebands are unstable unless there is sufficient Landau damping, such as the damping depicted in the left plot of Fig. 2. The opposite will be true below transition. The stability that we studied above is for a single azimuthal mode only, for example, either the upper or lower dipole synchrotron sideband in the language of only positive frequency. The spectrum of a bunch mode covers quite a number of synchrotron sidebands, some of which are stable and some unstable. To determine whether the bunch mode is stable or not, we need to sum up all the synchrotron sidebands of the bunch mode with $\eta \operatorname{Re} Z_{0}^{\|} / n$ as weights because all of them will be excited unless the impedance happens to vanish at those frequencies.

As an example, the growth rate of the $m$ th azimuthal mode can be expressed as

$$
\begin{aligned}
\frac{1}{\tau_{m}} & \propto \sum_{n=-\infty}^{\infty} \eta h_{m}\left(n \omega_{0}+m \omega_{s}\right) \frac{\operatorname{Re} Z_{0}^{\|}}{n}\left(n \omega_{0}+m \omega_{s}\right) \\
& =\sum_{n=0}^{\infty} \eta h_{m}\left(n \omega_{0}+m \omega_{s}\right)\left[\frac{\operatorname{Re} Z_{0}^{\|}}{n}\left(n \omega_{0}+m \omega_{s}\right)-\frac{\operatorname{Re} Z_{0}^{\|}}{n}\left(n \omega_{0}-m \omega_{s}\right)\right] .
\end{aligned}
$$

The power spectrum of the bunch $h_{m}(\omega)=\left|p_{m}(\omega)\right|^{2}$ enters into the expression with $p_{m}$ being the $m$ th azimuthal Fourier component of the linear bunch density because $p_{m}(\omega) Z_{0}^{\|}(\omega) / n$ gives the longitudinal wake field, which must be integrated over the bunch to get the total force. The first line of Eq. (5.1) addresses the upper sidebands associated with both positive and negative frequency, while the second line corresponds to folding the negative-frequency sidebands onto the positive-frequency sidebands. The second line therefore expresses the difference in longitudinal impedance at the upper and lower synchrotron sidebands flanking the positive revolution harmonic $n$. For a broadband impedance, this difference is close to zero. In other words, the contributions of the upper and lower sidebands flanking each revolution harmonic cancel each other even if there is no incoherent frequency spread. In the presence of incoherent frequency spread, Landau damping will lead to an additional amount of damping when all sidebands are summed. The conclusion is that, when coupling of different azimuthal modes is unimportant, a bunch is always stable if the driving impedance 
is broadband. This is quite different from the longitudinal instability of a coasting beam. Here, synchrotron oscillation plays an important role having the two components it creates, a growing and a damping, cancel each other. This conclusion does not exclude the possibility of the Keil-Schnell type of microwave instability for a coasting beam. This is because the bunch can be treated as a coasting beam when the wavelength of the instability is much less than the bunch length and when the growth time of instability is much smaller than the synchrotron oscillation period. In that case, there will not be any synchrotron sidebands established.

Equation (5.1), however, still leaves the option of a bunched-beam instability. The only possible instability that can occur in a bunch is when only one upper synchrotron sideband in Eq. (5.1) contributes essentially above transition (one lower sideband below transition.) This happens when the impedance is a narrow resonance of the width of or less than the synchrotron frequency leaning more towards an upper synchrotron sideband than the accompanied lower sideband and the other way around below transition. This explains why the fundamental mode of a rf cavity is usually detuned slightly downward from a revolution harmonic above transition and slightly upward below transition in order to guarantee stability. This is the so-called Robinson stability criterion [4] which manifests itself because of the fine tuning of the resonance frequency.

\section{Coherent Shift from Mean Incoherent Frequency}

Consider an impedance which has an inductive part and a resistive part and is well inside the stability region $(0<z<1)$ when the beam is above transition. The dynamic coherent synchrotron frequency shift must therefore be negative. However, without any synchrotron frequency spread, the dynamic coherent synchrotron frequency shift is positive for an inductive impedance. Is there a contradiction? Our conclusion is that in the absence of a synchrotron frequency spread, when the unperturbed distribution is elliptical, every particle has its synchrotron frequency shifted by the same amount and so is the center of the bunch. Therefore the static shift of the coherent synchrotron frequency is downward or $\Delta \omega_{s \text { coh }}^{\text {static }}=\Delta \omega_{s, \text { incoh }}=\omega_{s c}-\omega_{s 0}<0$. Since all particles execute synchrotron oscillation with exactly the same frequency, the bunch is rigid. The wake-field pattern moves with the bunch. Thus the motion of the bunch as a whole cannot be affected by the wake field at all. In other words, the dynamic coherent shift $\Delta \omega_{s \text { coh }}^{\text {dyn }}$ must be upward and is equal exactly 
to $\Delta \omega_{s \text { coh }}^{\text {static }}$. In the presence of a synchrotron frequency spread, the picture is different. The bunch will see the change in wake-field pattern and the bunch center will be affected by the wake. As a result, the coherent synchrotron frequency will be affected by the wake and the dynamic coherent synchrotron frequency shift will be different from $\Delta \omega_{s \text { coh }}^{\text {static }}$.

In the presence of a synchrotron frequency spread, the synchrotron frequency of the center-of-mass of the bunch due to the static effect of the impedance will be less than $\omega_{s c}$. This can be estimated by finding the rms of the synchrotron frequency of the individual particle because the synchrotron frequency appears as square in the equation of motion. We have

$$
\left\langle\omega_{s}^{2}\right\rangle=\int\left(\omega_{s c}-S r^{2}\right)^{2} \psi_{0} r d r d \theta
$$

For the elliptical distribution, the result is

$$
\left\langle\omega_{s}^{2}\right\rangle=\omega_{s c}^{2}\left[1-\frac{4}{5} \frac{S}{\omega_{s c}}+\frac{8}{35} \frac{S^{2}}{\omega_{s c}^{2}}\right],
$$

where the last term can be neglected because usually $S / \omega_{s c} \ll 1$. Thus, in the presence of a large spread, the synchrotron frequency of the center-of-mass is roughly $\omega_{s c}-\frac{2}{5} S$ from the static effect of the impedance. If $\Delta \omega_{s \text { dyn }} / S=\frac{1}{2}$, or the spread in synchrotron frequency is twice the static coherent shift without spread, the dispersion relation gives a dynamic coherent shift of $\Delta \omega_{s \text { coh }}^{\mathrm{dyn}}=-0.209 S$ according to Eq. (3.6). This shift is negative because it is measured from $\omega_{s c}$. However, this shift becomes $+0.191 S$, which is positive, when measured from $\omega_{s c}-\frac{2}{5} S$, the synchrotron frequency of the center-of-mass with the static effect included only.

On the other hand, $\Delta \omega_{s \text { dyn }}$ shifts from $\omega_{s c}$ in the negative direction when $\eta \operatorname{Im} Z_{0}^{\|} / n<0$ (dynamical coherent shift in the absence of spread). Now in the presence of an incoherent frequency spread $S$, the rms incoherent synchrotron frequency is no longer at $\omega_{s c}$, but becomes somewhere in between $\omega_{s c}$ and $\omega_{s c}-S$. Thus an additional coherent shift from the dynamical effects in the negative direction can easily land the coherent synchrotron frequency outside the incoherent frequency spread (more negative than $\omega_{s c}-S$ ). This may explain why there will not be any Landau damping when $\eta \mathcal{I} m Z_{0}^{\|} / n<0$. 


\section{Addendum}

It will be nice to repeat the above argument with the bunch in other distributions as well. However, the investigation becomes much more involved, because besides the incoherent synchrotron frequency spread coming from the sinusoidal rf potential which is intensity independent, there is now also the incoherent synchrotron frequency spread coming from the nonlinear longitudinal self-force which is intensity dependent. The general method to tackle the problem is to move this intensity dependent synchrotron frequency spread from the left side of Eq. (2.10) to the right side, so that the interaction matrix now consists of the original wake-force part plus the new synchrotron-frequency-spread part. Although the interaction matrix becomes more complex, however, the left side contains only the same synchrotronfrequency spread coming from the rf potential. Thus all investigation can proceed as before and we envision to arrive at the same conclusion as in the elliptical distribution.

As an addendum, we are going to give the stability threshold contours for the higher azimuthal modes as well as for other distributions in the longitudinal phase space. However, we shall ignore the incoherent synchrotron frequency spread due to nonlinear self-field effects, because their inclusion will lead to nonanalytic expressions.

\subsection{Higher Azimuthal Modes}

For higher azimuthal modes, such as the quadrupole modes $(m=2)$, sextupole modes $(m=3)$, etc. the dispersion relation can be derived in the same way as the dipole modes. Again we include only the most easily excited radial modes using synthetic kernels, which can be inferred easily from Eq. (2.11). Remembering that the $m$ th multipole kernel $K_{m}\left(r, r^{\prime}\right)$ is nothing more than the $m$ th multipole wake function, we must have

$$
K_{m}\left(r, r^{\prime}\right) \propto r^{m} r^{\prime m-1}
$$

The $m$ th multipole interaction matrix is therefore $G_{m}\left(r, r^{\prime}\right)=C_{m} r^{m} r^{\prime m-1}$ where $C_{m}$ is a constant. From the simplified Sacherer's integral equation in Eq. (2.10), it is easy to derive the dispersion relation,

$$
1=\frac{\Delta \omega_{m}}{W_{m}} \int \frac{\psi_{0}^{\prime}(r) r^{2 m}}{\Omega-m \omega_{s c}+m S r^{2}} d r
$$


where the spread $S$ in incoherent synchrotron frequency has been included and $C_{m}=$ $-\Delta \omega_{m} / W_{m}$. We have introduced

$$
W_{m}=\int_{0}^{\infty} \psi_{0}^{\prime}(r) r^{2 m} d r,
$$

so that in the absence of incoherent spread $(S=0), \Delta \omega_{m}=\Omega-m \omega_{s c}$ is the dynamic part of the $m$ th azimuthal coherent frequency shift. We further define

$$
z=\frac{\omega_{s c}-\Omega / m}{S}
$$

and change the variable of integration to $t=r^{2}$. The dispersion relation finally takes the form

$$
1=\frac{\Delta \omega_{m}}{m S W_{m}} \int_{0}^{\infty} \frac{\psi_{0}^{\prime}(t) t^{m}}{t-z} d r
$$

where we have used the short-hand notation $\psi_{0}^{\prime}(t) \equiv\left[d \psi_{0}(r) / d r^{2}\right]_{r^{2}=t}$. In above, $\Delta \omega_{m}=$ $C_{m} /(\pi S)$ with $\Delta \omega_{1}=\Delta \omega_{s \text { dyn }}$, the dynamic part of the coherent synchrotron frequency shift in the absence of an incoherent synchrotron frequency spread. It is convenient to define

$$
\mathcal{G}_{m}(t)=\frac{t^{m} \psi_{0}^{\prime}(t)}{m W_{m}}
$$

so that the dispersion relation simplifies to

$$
1=\frac{\Delta \omega_{m}}{S} \int_{0}^{\infty} \frac{\mathcal{G}_{m} t d t}{t-z} .
$$

The stability threshold curve can then be readily solved and is given by

$$
1=\frac{\Delta \omega_{m}}{S}\left[\mathcal{H}_{m}(z)-i \pi \mathcal{G}_{m}(z)\right]
$$

where

$$
\mathcal{H}(z)=\wp \int_{0}^{\infty} \frac{\mathcal{G}_{m}(t) d t}{t-z}
$$

For the elliptical distribution, it is easy to obtain the solution:

$$
\begin{aligned}
\mathcal{G}_{m}(z) & =-\frac{3 z^{m}}{4 \pi m W_{m} \sqrt{1-z}}, \\
\mathcal{H}_{m}(z) & =\frac{1}{m I_{m}}\left[\sum_{n=0}^{m-1} I_{m-n-1} z^{n}+\frac{z^{m}}{2 \sqrt{1-z}} \ln \left|\frac{\sqrt{1-z}+1}{\sqrt{1-z}-1}\right|\right], \\
W_{m} & =-\frac{3 I_{m}}{2 \pi} \quad \text { and } \quad I_{m}=\int_{0}^{1}\left(1-u^{2}\right)^{m} d u=\frac{2^{m} m !}{(2 m+1) ! !},
\end{aligned}
$$


when $0<|z|<1$, and analytic continuation elsewhere. The stability contours for the higher azimuthal modes are depicted in the right plot of Fig. 5. We see that the stable regions for higher azimuthal modes are much larger; especially, the point corresponding to $z=0$ is getting larger and larger. Physically, the point at $z=0$ (Point A in Fig. 2) is determined by the unperturbed distribution. If we consider Point A as the threshold, then the thresholds for various azimuthal modes can be computed by evaluating $\mathcal{H}(0)$. The principal-value integral can be performed easily to give

$$
\mathcal{H}(0)=\frac{I_{m-1}}{m I_{m}}
$$

The threshold for the $m$ th azimuthal mode is then

$$
\frac{\Delta \omega_{m}}{S}=\frac{m^{2}}{m+\frac{1}{2}},
$$

which gives $\frac{2}{3}$ for $m=1, \frac{8}{5}$ for $m=2, \frac{18}{7}$ for $m=3$, etc.

\subsection{Other distributions}

The elliptical distribution, when projected onto the rf phase axis $\tau$, results in the parabolic linear distribution, $\frac{3}{4}\left(1-\tau^{2}\right)$, which has a discontinuity in linear intensity gradient at the edges of the bunch, where we have set the half bunch length $\hat{\tau}=1$ for convenience. An improvement is the distribution

$$
\psi_{0}(r)=\frac{2}{\pi}\left(1-r^{2}\right) \quad 0<r<1
$$

The projection onto the rf phase axis is $\frac{8}{3 \pi}\left(1-\tau^{2}\right)^{3 / 2}$, which has a continuous linear intensity gradient at the edges of the bunch. However, the distribution itself in the longitudinal phase space still does not have a continuous radial gradient. To remedy this, we introduce the distribution

$$
\psi_{0}(r)=\frac{n+1}{\pi}\left(1-r^{2}\right)^{n} \quad 0<r<1 .
$$

for different larger values of $n$. As $n$ gets larger and larger, the distribution becomes clustered more and more around the origin. Thus to compare their stability contours, we should not use the synchrotron frequency spread $S$ from the center to the edge of the bunch. Instead we should employ the half-width-at-half maximum spread $S_{\text {ншнм }}$, which is easily found to be

$$
S_{\mathrm{HWHм}}=\left(1-2^{-1 / n}\right) S
$$


For the bi-Gaussian distribution

$$
\begin{aligned}
\psi_{0}(r) & =\frac{1}{2 \pi} e^{-r^{2} / 2}, \\
S_{\text {нWнм }} & =\sqrt{2 \ln 2} S_{\mathrm{rms}} .
\end{aligned}
$$

when $S_{\text {rms }}$ is the spread of the synchrotron frequency from the bunch center to particles at the rms bunch length.

The dipole $(m=1)$ stability threshold curves of these distributions are computed. Here, we plot them on top of each other for comparison in Fig. 6.

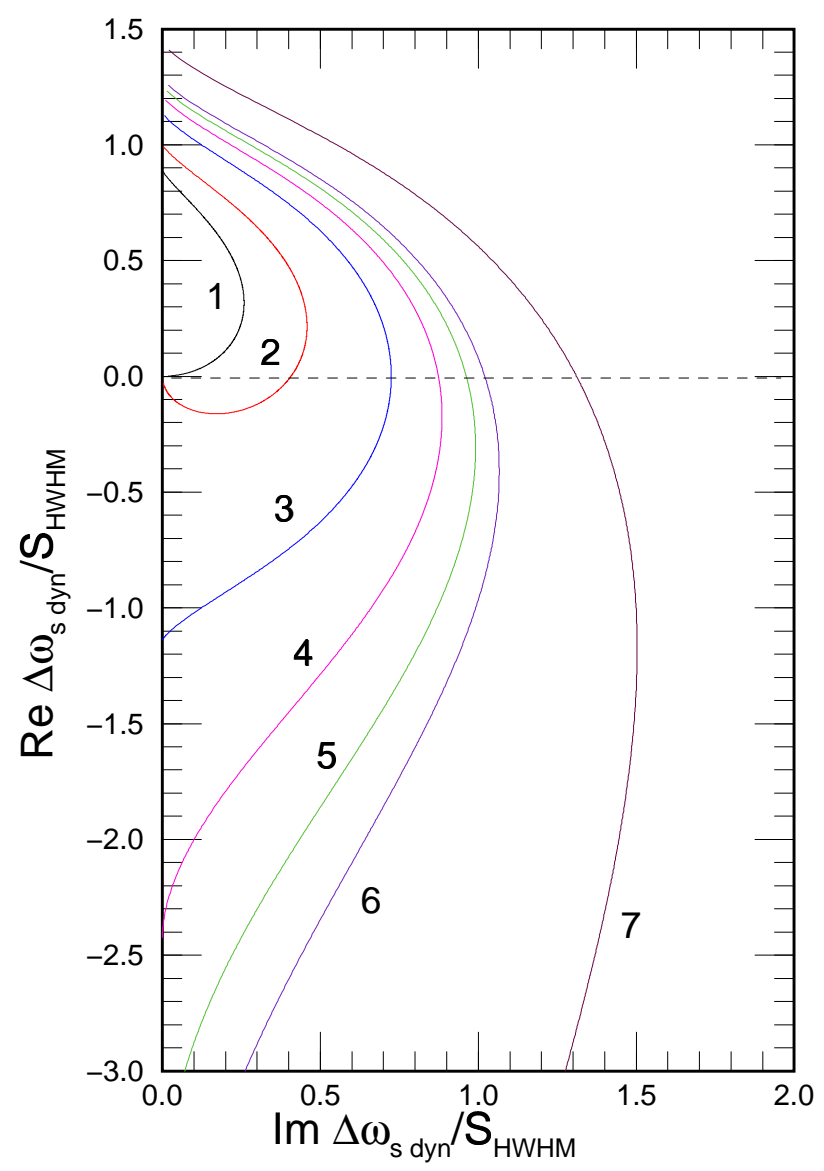

Figure 6: Stability threshold curves of the dipole mode $(m=1)$ for various distributions in order of smoother gradients at the edge of the bunch: (1) elliptical distribution $\psi_{0}(r)=\frac{3}{2 \pi} \sqrt{1-r^{2}}$, (2) $\frac{2}{\pi}\left(1-r^{2}\right)$, (3) $\frac{3}{\pi}\left(1-r^{2}\right)^{2}$, (4) $\frac{4}{\pi}\left(1-r^{2}\right)^{3}$, (5) $\frac{5}{\pi}\left(1-r^{2}\right)^{4},(6) \frac{5}{\pi}\left(1-r^{2}\right)^{5}$, (7) $\frac{1}{2 \pi} e^{-r^{2} / 2}$.

The analytic expressions for these stability contours are derived as follows. In fact, to draw these curves, it is only necessary to solve the integral in the region $0<z<1$. When 
the elliptical distribution is projected onto the phase axis, we have the parabolic distribution which entails an abrupt change of intensity gradient at the end of the bunch. In order to achieve a continuous intensity gradient, let us consider the distribution

$$
\psi_{0}(r)=\frac{2}{\pi}\left(1-r^{2}\right) \quad r \leq 1
$$

which results in the linear distribution $\frac{8}{3 \pi}\left(1-\tau^{2}\right)^{3 / 2}$. Here we have

$$
W_{m}=-\frac{2}{(m+1) \pi} \text {, }
$$

with

$$
\mathcal{G}_{m}=\frac{m+1}{m} t^{m}
$$

The threshold curves are given by

$$
\mathcal{H}_{m}(z)=\frac{m+1}{m}\left[\sum_{n=0}^{m-1} \frac{z^{n}}{m-n}+z^{m} \ln \left|\frac{1-z}{z}\right|\right] \text {. }
$$

These stability curves in the $\Delta \omega_{s \text { dyn }} / S_{\text {ншнм }}$-plane are shown in the right plot of Fig. 7 . On the left plot we show once more, for comparison those threshold contours of the elliptical distribution. This is essentially the same as the right plot in Fig. 5 with the exception that the HWHM spread $S_{\text {ншнм }}$ is used instead of the full spread.

For the distribution

$$
\psi_{0}(r)=\frac{3}{\pi}\left(1-r^{2}\right)^{2} \quad r \leq 1
$$

which results in the linear distribution $\frac{16}{5 \pi}\left(1-\tau^{2}\right)^{5 / 2}$, we have

$$
W_{m}=-\frac{6}{(m+1)(m+2) \pi}
$$

with

$$
\mathcal{G}_{m}=\frac{(m+1)(m+2)(1-t) t^{m}}{m} .
$$

The principal-value integral is very similar to that for the previous distribution. It is useful to introduce a reduced function $h_{m}(z)$ by

$$
h_{m}(z)=\wp \int_{0}^{1} \frac{t^{m} d t}{t-z}=\sum_{n=0}^{m-1} \frac{z^{n}}{m-n}+z^{m} \ln \left|\frac{1-z}{z}\right| .
$$

The function $H_{m}(z)$ of this distribution can now be written as

$$
\mathcal{H}_{m}(z)=\frac{(m+1)(m+2)}{m}\left[h_{m}(z)-h_{m+1}(z)\right]
$$



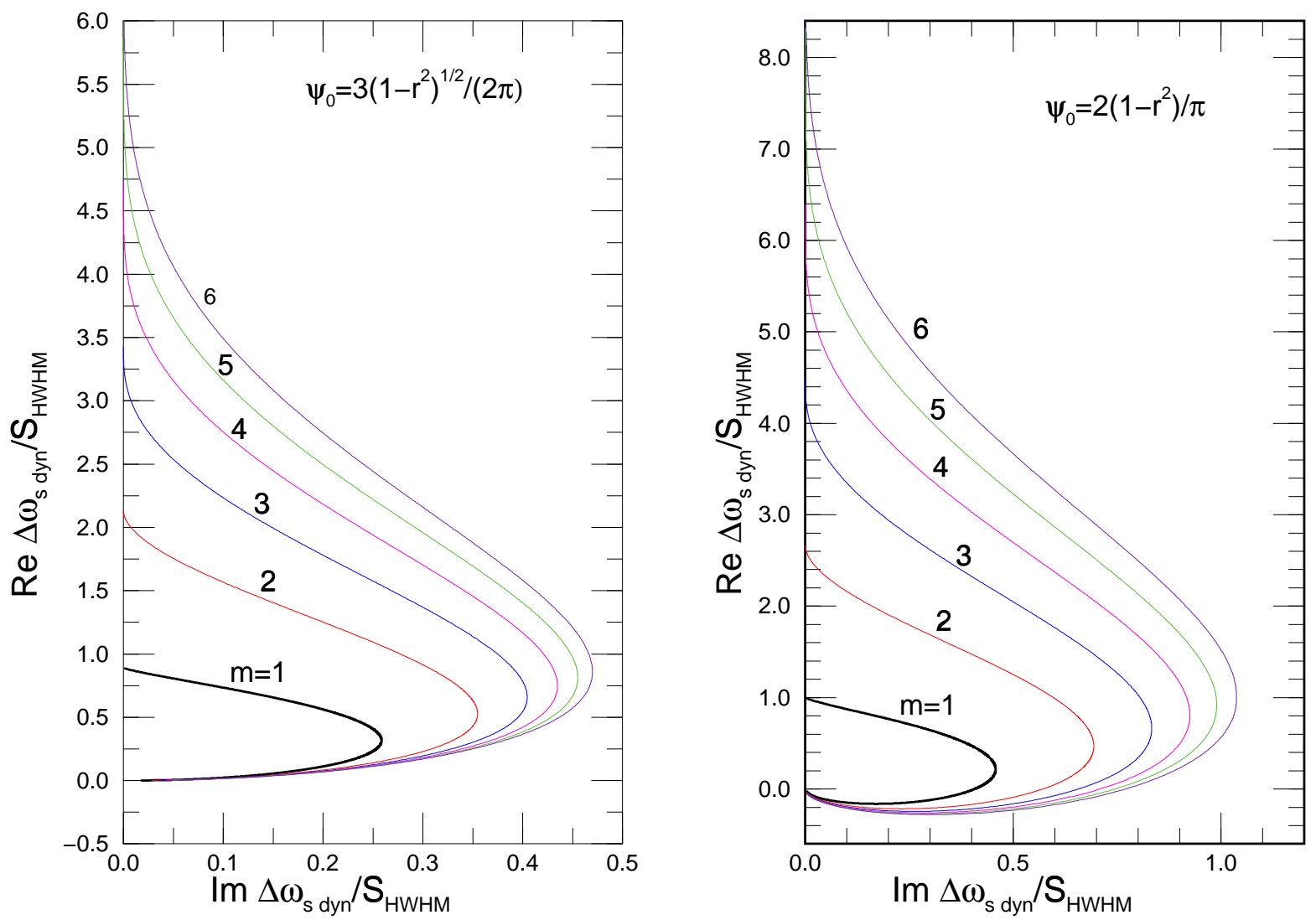

Figure 7: Stability threshold curves in the $\Delta \omega_{s \text { dyn }} / S_{\text {ншнм }}$-space for the lower azimuthal modes $(m=1,2, \cdots, 6)$ for (left) the elliptical distribution $\psi_{0}(r)=\frac{3}{2 \pi} \sqrt{1-r^{2}}$ and (right) $\psi_{0}(r)=\frac{2}{\pi}\left(1-r^{2}\right)$. The stability limits for $\operatorname{Re} \Delta \omega_{s \text { dyn }} / S_{\text {ншнм }}$ are, respectively, $\frac{4}{3} \frac{m^{2}}{m+\frac{1}{2}}$ and $\frac{\sqrt{2} m^{2}}{m+1}$.

In general, for the distribution

$$
\psi_{0}(r)=\frac{n+1}{\pi}\left(1-r^{2}\right)^{n},
$$

where $n$ is an integer, we have the general expression

$$
\mathcal{H}_{m}^{(n)}(z)=\frac{(m+n) !}{m m !} \sum_{k=0}^{n-1} \frac{(-1)^{k} h_{m+k}(z)}{k !(n-k-1) !},
$$

where the superscript $(n)$ denotes the power $n$ in the distribution. The stability limit is

$$
\operatorname{Re} \frac{\Delta \omega_{s \text { dyn }}}{S_{\text {нWнм }}}=\frac{1}{1-2^{-1 / n}} \frac{1}{\mathcal{H}_{m}^{(n)}(0)}=\frac{1}{1-2^{-1 / n}} \frac{m^{2}}{m+n},
$$

where the factor $1-2^{-1 / n}$ converts the HWHM frequency spread to the frequency spread at half width of the bunch. It is worth mentioning that Eq. (7.29) applies for the elliptical 
distribution also with $n=\frac{1}{2}$. For completeness, the threshold curves for $n=2,3$, 4, and 5 are plotted in Figs. 8 and 9.
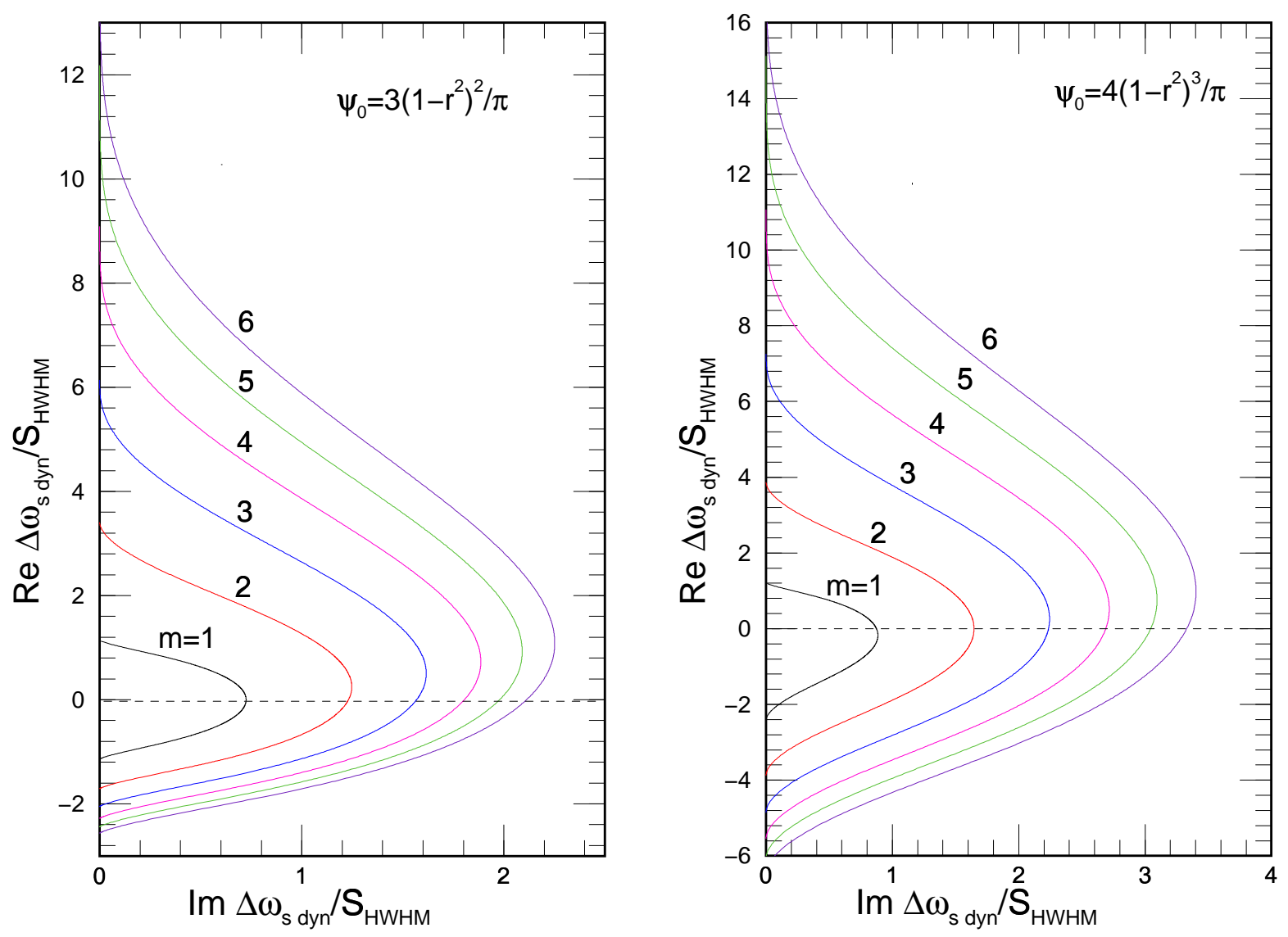

Figure 8: Stability threshold curves in the $\Delta \omega_{s \text { dуn }} / S_{\text {ншнм }}$-space for the lower azimuthal modes $(m=1,2, \cdots, 6)$ for distributions $\psi_{0}(r)=\frac{n+1}{\pi}(1-$ $\left.r^{2}\right)^{n}$ with $n=2$ on the left and $n=3$ on the right. The stability limits for $\operatorname{Re} \Delta \omega_{s \text { dyn }} / S_{\text {нwнм }}$ are $\frac{1}{1-2^{-1 / n}} \frac{m^{2}}{m+n}$.

\subsection{Bi-Gaussian Distribution}

The stability contours for any unperturbed distribution $\psi_{0}$ in the longitudinal phase space can be derived from the dispersion relation depicted in Eq. (7.5). Here, let us study the bi-Gaussian distribution

$$
\psi_{0}(r)=\frac{1}{2 \pi} e^{-r^{2} / 2}
$$



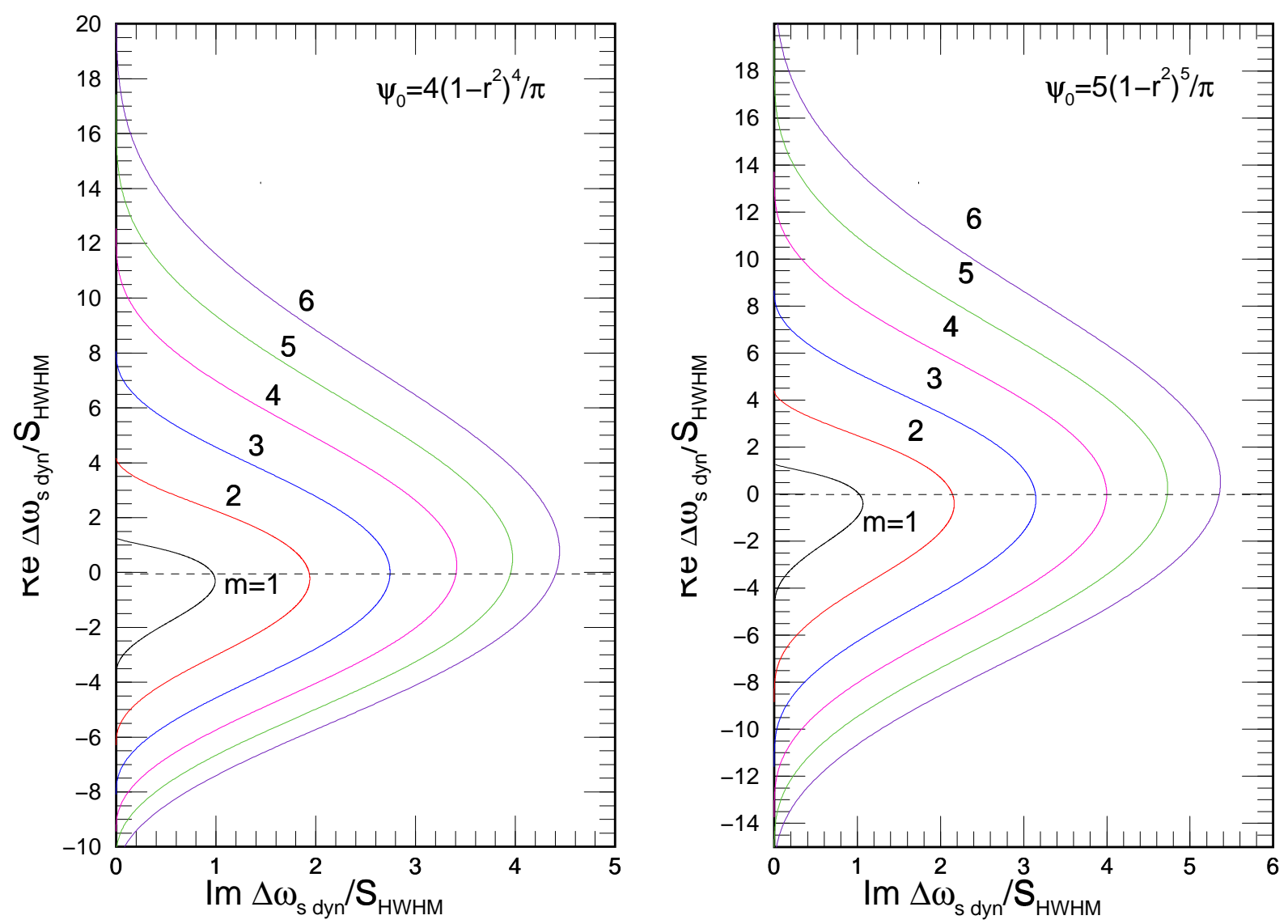

Figure 9: Stability threshold curves in the $\Delta \omega_{s \text { dyn }} / S_{\text {ншнм}}$-space for the lower azimuthal modes $(m=1,2, \cdots, 6)$ for distributions $\psi_{0}(r)=\frac{n+1}{\pi}(1-$ $\left.r^{2}\right)^{n}$ with $n=4$ on the left and $n=5$ on the right. The stability limits for $\operatorname{Re} \Delta \omega_{s \mathrm{dyn}} / S_{\mathrm{HWHM}}$ are $\frac{1}{1-2^{-1 / n}} \frac{m^{2}}{m+n}$.

where the rms spread of the bunch has been normalized to unity. The solution is given by the same expression in Eq. (7.8) with $S$ equal to the rms incoherent frequency spread, and

$$
\begin{aligned}
\mathcal{G}_{m}(z) & =\frac{z^{m} e^{z / 2}}{2^{m+1} m m !}, \\
\mathcal{H}_{m}(z) & =\frac{1}{2 m m !}\left[\sum_{n=0}^{m-1}(m-n-1) !\left(\frac{z}{2}\right)^{n}+\left(\frac{z}{2}\right)^{m} e^{-z / 2} E_{1}\left(-\frac{z}{2}\right)\right],
\end{aligned}
$$

where

$$
W_{m}=-\frac{2^{m} m !}{2 \pi}
$$

and

$$
E_{1}(u)=\int_{u}^{\infty} \frac{e^{-t} d t}{t}
$$



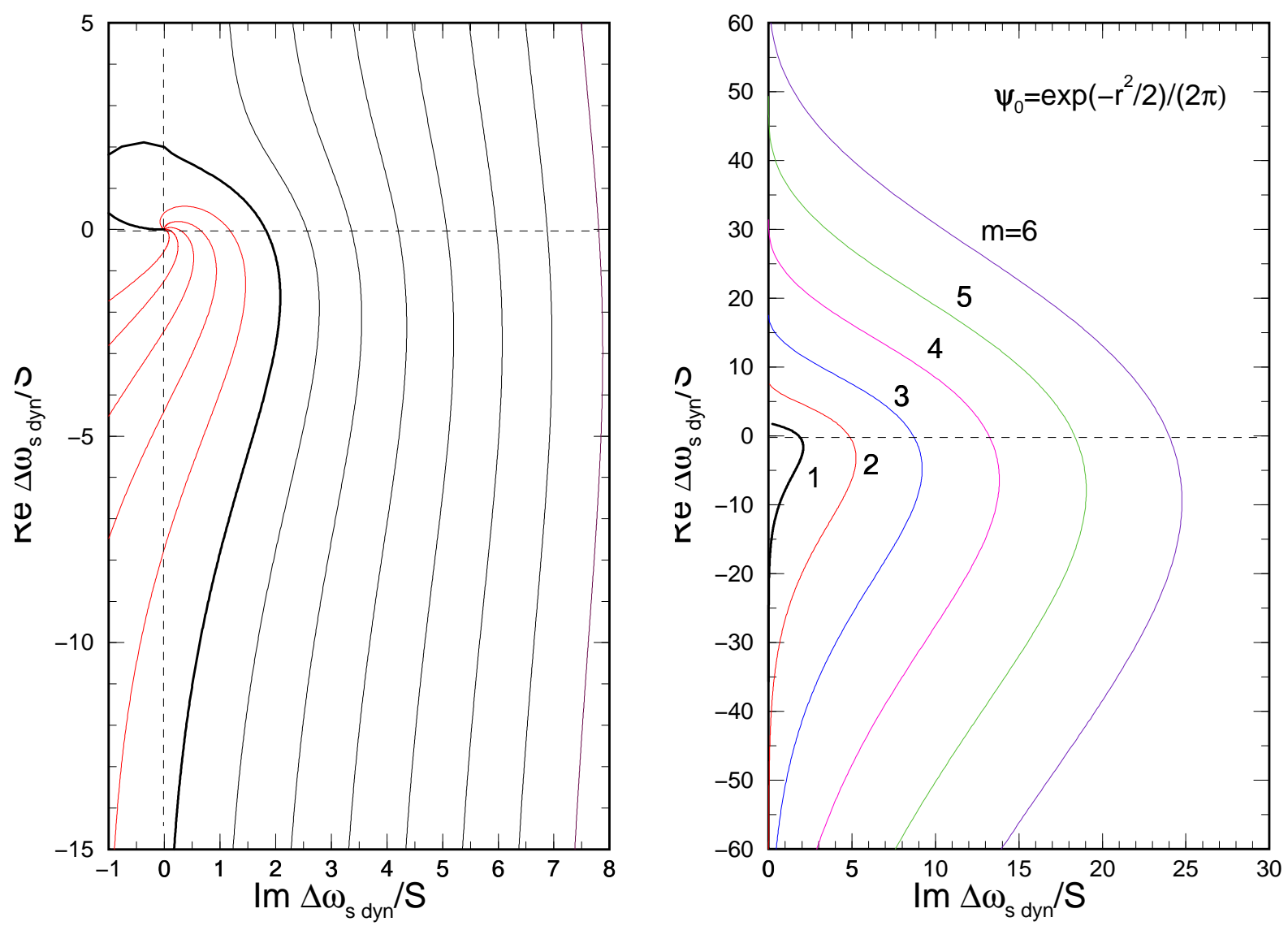

Figure 10: Left: Equi-Growth contours of the bi-Gaussian distribution $\psi_{0}(r)=e^{-r^{2}} /(2 \pi)$, with growth rate increasing by steps of $0.1 S$ to the left of the stability contour (darker curve) and damping rate increasing by steps of $0.1 S$ to the right, where $S$ is the rms spread of the incoherent synchrotron frequency. Right: Stability contours for the bi-Gaussian distribution for azimuthal modes $m=1$ to 6 .

is the exponential integral. The stability contour and equi-growth contours are depicted in the left plot of Fig. 10 with growth rates in steps of $S$. In the right plot, we show the stability contours for the higher azimuthal modes. 


\section{References}

[1] F.J. Sacherer, A Longitudinal Stability Criterion for Bunched Beams, CERN report CERN/MPS/BR 73-1, 1973; IEEE Trans. Nuclear Sci. NS 20, 3, 825 (1973).

[2] B. Zotter, Longitudinal Stability of Bunched Beams Part II: Synchrotron Frequency Spread, CERN Report CERN SPS/81-19 (D1), 1981.

[3] F.J. Sacherer, Methods for Computing Bunched-Beam Instabilities, CERN Report CERN/SI-BR/72-5, 1972.

[4] P.B. Robinson, Stability of Beam in Radiofrequency System, Cambridge Electron Accel. Report CEAL-1010, 1964. 